\title{
Patrones de dispersión de semillas y colonización de Misodendrum punctulatum (Misodendraceae) en un matorral postfuego de Nothofagus antarctica (Nothofagaceae) del noroeste de la Patagonia
}

\author{
Misodendrum punctulatum (Misodendraceae) seed dispersal and colonization patterns on \\ a Nothofagus antarctica (Nothofagaceae) post-fire shrubland from Northwestern \\ Patagonia
}

\author{
NORLAN TERCERO-BUCARDO* \& ADRIANA E. ROVERE
}

Laboratorio Ecotono-Centro Regional Universitario Bariloche, Universidad Nacional del Comahue, Quintral 1250, San Carlos de Bariloche (8400), Río Negro, Argentina. Consejo Nacional de Investigaciones Científicas y Técnicas

*Autor correspondiente: ntbucardo@gmail.com

\begin{abstract}
RESUMEN
El patrón espacial de la dispersión puede afectar el patrón espacial de establecimiento condicionando la estructura de la vegetación. Este trabajo analiza la magnitud de la dispersión y la colonización (infección) de la planta hemiparásita Misodendrum punctulatum (Misodendraceae). Este estudio fue realizado en un matorral postfuego de Nothofagus antactica (hospedador). Nuestra hipótesis plantea que el patrón de dispersión anemófila de las semillas de $M$. punctulatum condiciona el patrón de infección (colonización) sobre $N$. antarctica y que ambos procesos (dispersión y colonización) son asimétrico en la misma dirección en función del viento predominante. Se evaluó la dispersión a campo contando el número de semillas sobre troncos y ramas desde arbustos remanentes infectados (hospedadores focales) hacia cada punto cardinal. La colonización se evaluó registrando el número de infecciones sobre cada hospedador a lo largo de franjas trazadas hacia cada orientación desde los arbustos focales. Se observó que la cantidad de semillas dispersadas declina con la distancia desde la fuente de semillas, ajustándose a una distribución exponencial negativa. La mayor distancia de dispersión primaria fue de $10 \mathrm{~m}$ hacia el este. Respecto a la colonización la mayor distancia de nuevos establecimientos fue $22.5 \mathrm{~m}$ en dirección sur. Por otro lado, el número de infecciones desde los arbustos focales fue menor al oeste (1 infectado: 10 no infectados) que en las otras direcciones (1 infectado: 6 no infectados). Ambos procesos presentaron un patrón de distribución similar y asimétrica como consecuencia de la dirección de los vientos predominantes y se observó una alta correlación espacial entre la dispersión de semillas y el establecimiento de las infecciones. Estos resultados de dispersión y colonización permiten comprender los patrones de distribución espacial de esta planta hemiparásita y entender su dinámica de infección. Los resultados de este trabajo podrían ser útiles en proyectos de restauración ecológica, por ejemplo para la reintroducción de $M$. puctulatum sobre ejemplares de $N$. antarctica que rebrotan en matorrales postfuegos. Estos aspectos estudiados también aportan al conocimiento de esta interacción ecológica que ocurre frecuentemente en los bosques de Nothofagus de Argentina y Chile.
\end{abstract}

Palabras clave: dinámica, dispersión anemófila, hospedador, parasitismo, sucesión.

\begin{abstract}
Spatial dispersal pattern could determine the spatial pattern of establishment affecting the vegetation structure. Our work, analyze dispersion and colonization attribute of the hemiparasitic plant Misodendrum punctulatum (Misodendraceae). Our hypothesis outline that the wind dispersal pattern of $M$. punctulatum seeds determine the infection pattern (colonization) on Nothofagus antarctica, and both processes (dispersion and colonization) are asymmetric in accordance with the predominant wind direction. This study was carry out in a $N$. antarctica (host) post-fire shrubland. We assessed the seed dispersal estimating number of seed on host branches supplied from infected-survivals hosts (focal host) toward each cardinal direction. Colonization was assessed estimating number of infections on each host, through transects, at every cardinal direction from the focal host. We observed that seeds dispersed decline with distances from the seed source, and dispersion fitted to a negative exponential model. The longest distance of primary dispersion observed was $10 \mathrm{~m}$ toward east. About colonization, longest distance from the focal host was $22.5 \mathrm{~m}$ toward south. Furthermore, ratio of infected host was lesser on west, one infected by 10 uninfected (1:10) than other compass aspects (1:6). Both process, dispersion and colonization, showed a similar and asymmetric pattern of distribution, which could be result of wind direction predominant from northwest. In addition, a high spatial correlation was observed among number of seed dispersed and recruitment of infection in distances from the sources of seeds. Our results allow
\end{abstract}


understand the patterns of spatial distribution and infection dynamics of Misodendrum punctulatum. In order to plan management strategies, understanding the infection dynamics is useful to perform successfully strategies to the control of hemiparasitic plants in forest to commercial use, and conservation or restoration strategies, e.g. to restorate this hemiparasitic specie in areas recently colonized by $N$. antarctica. These studied aspects in Misodendrum punctulatum contribute to the knowledge of this ecological interaction, which is frequent in Nothofagus forests from Argentina and Chile.

Key words: dynamics, host, parasitism, succession, wind dispersion.

\section{INTRODUCCIÓN}

Las plantas parásitas representan aproximadamente $1 \%$ del total de las especies angiospermas, con más de 3000 especies distribuidas en 16 familias (Agrios 1969, Norton \& Carpenter 1998). La mayor parte de los estudios sobre plantas parásitas focalizan en la ecología de la frugivoría y solo recientemente se ha incorporado la perspectiva parásitohospedero (Medel et al. 2002). Esta perspectiva evalúa cuantitativamente los determinantes históricos y biogeográficos de esta interacción planta-planta, la autoecología de la biología floral, polinización, dispersión, estrategias de colonización de las parásitas y mecanismos de defensa de los hospedadores. Con respecto a la dispersión, distintos trabajos han analizado la interacción mutualista de la frugivoría, ya que la mayoría de ellos son dispersados por aves (Calder 1983), mamíferos (Amico \& Aizen 2000), y mecanismos balísticos de autodispersión y epizoocoria (Hawksworth 1961, Agrios 1969, Hawksworth \& Wiens 1996, Restrepo et al. 2002). Contrariamente, la dispersión anemófila en las plantas parásitas es poco común (Orfila 1976, 1978). De hecho, los únicos géneros dispersados por el viento son Nuytsia (Lorantaceae) (Lamont 1985) y Misodendrum (Orfila 1976, 1978).

Los mecanismos de dispersión de semillas, desempeñan una función central en la dinámica poblacional y comunitaria de las plantas (Greene \& Johnson 1989, Wang \& Smith 2002). La dispersión de semillas es la fase demográfica más importante para el movimiento de las plantas en el espacio, dado que todas las especies en estado sucesional desaparecerán de su hábitat actual y su continua supervivencia depende del escape y el establecimiento (Harper 1977). Este desplazamiento delimita el área potencial de reclutamiento y afecta los procesos de depredación y competencia, lo cual eventualmente resultará en un nuevo patrón espacial de individuos capaces de reproducirse
(Nathan et al. 2001). Como resultado de la dispersión primaria desde la planta madre, las semillas quedan distribuidas de forma heterogénea, generando un patrón de distribución espacial denominado sombra de semillas (Willson 1992). La sombra de semillas hace referencia al patrón de distribución espacial de la dispersión desde una planta, o desde una fuente de semillas compuesta por múltiples plantas, y se caracteriza por una alta concentración de semillas cerca de la fuente. En la mayoría de las plantas, la sombra de semillas existe en dos dimensiones horizontales, con excepción de las plantas epifitas y las plantas parásitas aéreas, en las cuales se incorpora una tercera dimensión vertical (Willson 1992, Overton 1994). Este patrón espacial de dispersión tendría una relación sinérgica con el patrón de reclutamiento o establecimiento condicionando la estructura espacial de la vegetación (Herrera et al. 1994, Wang \& Smith 2002). Sin embargo, la conexión entre estos dos patrones no es tan directa debido a que los requerimientos para la germinación y establecimiento pueden ser muy específicos en algunas especies. La germinación y establecimiento en plantas parásitas pueden ser mediado por señales químicas de hospederos específicos (Calder \& Bernhardt 1983, Rodl \& Ward 2002), y por ello solamente se las encuentran en lugares particulares (Willson 1992). En el caso particular de las plantas hemiparásitas, para poder germinar y establecerse es indispensable que la semillas lleguen hasta la especie hospedadora adecuada (McKinney \& Hemmerly 1977, Reid \& Lange 1988, Norton \& De Lange 1990, Monteiro et al. 1992, Overton 1994, Overton 1997, Norton \& Carpenter 1998, Tercero-Bucardo 2001, Medel et al. 2002). Es decir en estas plantas, no solo es importante la eficiencia del vector dispersor sino que también la efectividad del mismo. Reid (1989) y Bustamante \& Canals (1995) se refieren a la eficiencia de dispersión como la probabilidad de que las semillas dispersadas 
por un vector alcancen un sitio seguro para germinar, y definen la efectividad como la proporción de plántulas de una población de plantas que un vector es responsable de diseminar. A su vez, habiendo llegado a la especie hospedadora adecuada, el éxito de establecimiento puede estar condicionado por características físicas de los hospedadores como el diámetro de la rama, el grosor de la corteza y otras defensas que el hospedador haya desarrollado (Reid 1989, Sargent 1995, Tercero-Bucardo \& Kitzberger 2004).

La dispersión anemócora de especies del bosque templado austral de Argentina y Chile ha sido escasamente estudiada (pero véase Kitzberger 1994, Bustamante 1996, TerceroBucardo 2001, Rovere \& Premoli 2005), a pesar de que el $47 \%$ de los géneros australes de Sudamérica son dispersados por agentes abióticos (Aizen \& Ezcurra 1998). El objetivo del presente trabajo es describir cuantitativamente el patrón espacial de dispersión de semillas y colonización de la especie de plantas hemipárasitas Misodendrum punctulatum Banks ex DC. Nuestra hipótesis es que el patrón de dispersión anemófila de las semillas de $M$. punctulatum condiciona el patrón de infección (colonización) sobre Nothofagus antarctica (G. Forst.) Oerst y que ambos procesos (dispersión y colonización) son asimétricos en la misma dirección en función del viento predominante. Los resultados de este trabajo contribuirán al conocimiento de la dinámica de dispersión e infección de $M$. punctulatum en matorrales postfuego de los bosques templados del sur de Sudamérica. Asimismo, permitirán comprender los patrones de distribución espacial de esta especie hemiparásita y los factores que los determinan. Para fines de manejo, conocer la dinámica de infección de Misodendrum puede ser útil para planificar estrategias de control en rodales de interés comercial. También para proyectar la incorporación de esta especie con fines de restauración ecológica en áreas degradadas.

\section{MÉTODOS}

\section{Área de estudio}

Este trabajo se realizó en un matorral postfuego de $N$. antarctica, en un incendio ocurrido aproximadamente en el año 1973 en un fondo de valle próximo al lago

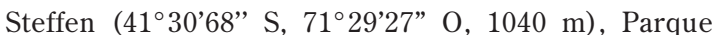
Nacional Nahuel Huapi. Nothofagus antarctica regenera por rebrote y ocupa entre el $80-90 \%$ de la cobertura vegetal en este sitio y no está asociada a otras especies de Nothofagus. Como especies acompañantes también se encuentran Diostea juncea, Lomatia hirsuta, Berberis buxifolia y Schinus patagonica. Los vientos predominantes son del noroeste, la precipitación anual del sitio es de $1200 \mathrm{~mm}$, en esta zona llueve principalmente otoño e invierno (abril-septiembre), en forma de nieve o lluvia, mientras que los meses de verano son relativamente secos y cálidos (diciembremarzo) (De Fina 1972). La temperatura promedio mensual es de aproximadamente $2^{\circ} \mathrm{C}$ y $14^{\circ} \mathrm{C}$ en verano.

\section{La especie parásita}

Misodendrum punctulatum Banks ex DC. (Misodendraceae) es una planta hemiparásita, comúnmente llamada farolito chino o flor de ñire, es una especie dioica, pertenece a una familia monogenérica endémica de los bosques del sur de Argentina y Chile. La familia Misodendraceae comprende a ocho especies que infectan específicamente a las especies del género Nothofagus sobre todo su rango de distribución geográfico $\left(33^{\circ}\right.$ $56^{\circ}$ S) (Orfila 1976, Rossow 1982). En los bosques andino-patagónicos de Nothofagus del lado argentino $M$. punctulatum es una de las especies más abundantes del género, y en Argentina y Chile su distribución coincide con la del género Nothofagus (Orfila 1978). Misodendrum punctulatum, es parásita principalmente de $N$. pumilio (Opep. \& Ende.) Krasser (lenga), $N$. antarctica (G. Forst.) Oerst (ñire) y N. dombeyi (Mirb.) Oerst (coihue). Los frutos de $M$. punctulatum son aquenios secos y pequeños $(2 \mathrm{~mm})$ provistos de tres setas de hasta $1 \mathrm{~cm}$ de longitud (Orfila 1976) que les permiten volar y anclarse pasivamente de manera principal en pequeñas ramas y tallos del hospedador. Los tallos de $M$. punctulatum presentan crecimiento simpodial (Orfila 1976, 1978), poseen tejidos fotosintéticos reducidos con hojas en forma de pequeñas escamas. Después de la dispersión las semillas germinan sobre las ramas de los hospedadores, el hipocótilo recurvado crece y se adhiere a la rama hospedadora a través de substancias pegajosas. Finalmente el haustorio (hipocótilo modificado) penetra el xilema del árbol estableciendo la relación hospedador-hemiparásita. Misodendrum punctulatum florece durante la primavera (desde finales de septiembre a inicios de noviembre), sus frutos maduran al inicio del verano, la dispersión ocurre entre enero y febrero y las semillas germinan durante la siguiente primavera (Tercero-Bucardo 2001, Tercero-Bucardo \& Kitzberger 2004). Se ha observado que las infecciones de ramas pueden vivir hasta 22 años y más de 100 años cuando se establecen en los troncos principales del hospedador, esto define a $M$. punctulatum como una de las plantas hemiparásitas más longevas del mundo, reportadas hasta ahora (Tercero-Bucardo \& Kitzberger 2004).

\section{La especie hospedadora}

Nothofagus antarctica (G. Forst.) Oerst., comúnmente llamada ñire o ñirre, presenta la mayor amplitud ecológica de las especies de Nothofagus sudamericanos (Donoso et al. 2006). Es una especie caducifolia que puede encontrarse tanto como árboles de 10 a $15 \mathrm{~m}$ de alto en sitios con condiciones óptimas o como arbustos 
de 1 a $3 \mathrm{~m}$ en sitios xéricos, rocosos o expuestos a condiciones adversas (Veblen et al. 1996). Esta es considerada una especie pionera al igual que otras especies de Nothofagus, ya que es intolerante a la sombra y posee cierta adaptabilidad a las condiciones de campo abierto (Donoso 1993), a la vez que presenta una corta dispersión anemócora de sus semillas (Knapp et al. 2005). En los bosques del norte de la Patagonia, $N$. antarctica se convierte en la especie dominante inmediatamente después del fuego, esto hace que se vea favorecida en áreas afectadas por incendios frecuentes (Veblen \& Lorenz 1987). Posee una capacidad de reproducción vegetativa mayor que otras especies de Nothofagus, lo cual constituye un modo de propagación para la especie especialmente en relación a la ocurrencia de disturbios. Su dinámica está fuertemente asociada a incendios forestales y es ramoneada por herbívoros ungulados (Veblen et al. 1996). Esta especie, también se reproduce fácilmente en forma asexual en condiciones naturales (Donoso et al. 2006). Produce rebrotes a partir de raíces adventicias desde ramas parcialmente enterradas y regenera vigorosamente desde la base del tronco (Veblen et al. 1996, Vidal-Russell \& Premoli 2004). En comparación a las otras especies del genero tiene vida corta, mostrando signos de senescencia entre los 80120 años (Veblen et al. 1996). Misodendrum punctulatum crece prolíficamente sobre $N$. antarctica, favoreciendo el debilitamiento de sus ramas y el ataque por otros agentes de daño (Donoso et al. 2006). La infección (establecimiento) de esta hemiparásita en las ramas del hospedador produce tumores, también efectos negativos en la calidad de la madera (Reyes et al. 1986)

\section{Dispersión de semillas}

Los estudios de dispersión de semillas en bosques se hacen en general a partir de plantas aisladas o plantas ubicadas en los bordes de bosques (Harper 1977, Rovere \& Premoli 2005). El muestreo de dispersión de semillas de $M$. punctulatum se realizó al finalizar el período de dispersión (a finales de enero) en una población de $N$. antarctica. Para ello se eligieron 10 arbustos de $N$. antarctica (hospedador) los cuales contenían infecciones individuales (infección focal) que eran plantas femeninas de $M$. punctulatum, de tamaño similar, en etapa reproductiva. Cada arbusto remanente estaba aislado al menos $50 \mathrm{~m}$ de otros ejemplares infectados. El muestreo se realizó en franjas de $20 \mathrm{~m}$ de largo por $1 \mathrm{~m}$ de ancho en dirección a cada punto cardinal, i.e. se realizaron cuatro franjas por hospedador. Dentro de cada franja se registró el número de semillas encontradas tanto en las ramas del hospedador que contenía a la infección focal como de potenciales hospedadores no infectados presentes en el área muestreada. También se registró la distancia desde infección focal a cada rama donde se encontraban las semillas. Esta metodología utilizada para medir semillas, por medio de observaciones directas o censos visuales sobre el piso del bosque a lo largo de franjas, fue utilizada antes por otros autores (Cerdeira Morellato \& Voltolini $2000^{1}$ ). La distancia a

1 CERDEIRA MORELLATO LP \& VOLTOLINI JC (2000) Field methods to estimate fruit and seed production. 3rd International SymposiumWorkshop on Frugivores and seed dispersal biodiversity and conservational perspectives. August 6-11, 2000. São Pablo, Brasil. lo largo de la cual se evaluó la dispersión en nuestro trabajo, se calculó basándose en la relación utilizada como norma en silvicultura, donde la distancia de diseminación por viento de las semillas en especie forestales varía entre una y dos veces la altura del árbol (Donoso 1993). Dado que la altura de los arbustos hospedadores en el sitio de estudio era de 3 a $6 \mathrm{~m}$ y copas de aproximadamente $3 \mathrm{~m}$ de diámetro, la distancia en la cual se observó la dispersión de semillas de $M$. punctulatum cubre más de lo exigido por dicha norma.

\section{Colonización}

La colonización de $M$. punctulatum sobre $N$. antartica se evaluó en el mes de enero en el mismo matorral postfuego donde se estudió la dispersión. El patrón de colonización se determinó a partir de 16 arbustos focales de $N$. antartica, remanentes postfuego, los cuales tenían más de 10 infecciones de $M$. punctulatum (plantas femeninas y masculinas). Los mismos se encontraban como mínimo a $50 \mathrm{~m}$ distantes entre sí y ubicados aproximadamente a $300 \mathrm{~m}$ del fragmento más cercano de bosque no quemado. Se trazó una franja de $30 \mathrm{~m}$ desde cada arbusto focal, se registró la distancia de los arbustos de $N$. antartica colonizados por nuevas infecciones (distancia de recolonización). Cada una de las 16 franjas fue colocada al azar según uno de los puntos cardinales hasta completar cuatro para cada dirección.

\section{Análisis de datos}

La altura de los arbustos indica la altura promedio desde donde ocurre la dispersión, ya que algunas de las hemiparásitas pueden estar infectando ramas altas o ramas bajas. Para calcular las distancias de dispersión se promediaron las distancias obtenidas para los distintos hospedadores, a fin de evitar que las diferencias pudieran deberse a la altura de la planta (Willson 1992). Es importante mencionar que en este trabajo la medición de la distancia de dispersión corresponde a un único evento, por el contrario la colonización (infección) puede representar varios eventos, ya que es el resultado de lo acumulado durante varios años. También es importante aclarar que el muestreo podría estar subestimando el número de infecciones, debido a que se sabe que las plantas de $M$. punctulatum, al inicio de su ciclo de vida, tienen un período variable de (2-3 años) durante el cual están presentes pero como "holoparásitas", i.e. solo crece internamente dentro del tejido del hospedador, sin producir ramas (Tercero-Bucardo \& Kitzberger 2004).

A partir de los datos registrados, se calculó el número de semillas dispersadas hacia cada punto cardinal a intervalos de $2 \mathrm{~m}$ desde el hospedador focal. Se utilizó dicho rango $(2 \mathrm{~m})$ ya que el diámetro promedio de la copa de los hospedadores era de $3 \mathrm{~m}$, de esta manera la primera categoría $(0-2 \mathrm{~m})$ agruparía a las semillas que caen dentro del hospedador focal que alberga a la fuente de semillas. A partir de estos datos de dispersión se estimó la forma de la curva de distribución de las semillas para cada orientación (norte, sur, este y oeste), también para el promedio de todas las orientaciones. Mediante un paquete estadístico de ajuste de funciones, se realizaron pruebas estadísticas de la bondad de ajuste de los datos a un modelo y se obtuvieron los parámetros del modelo que mejor explicaba la distribución de los datos. Con los valores promedios de número de semillas y número de 
infecciones para cada distancia y orientación, se realizaron análisis pareados por orientación mediante la prueba de Wilcoxon. Mediante correlación de Sperman se estimó la relación entre el promedio del número de semillas y el promedio de las infecciones para cada distancia. También se realizó el test de KolmogorovSmirnov a fin de comparar la distribución promedio (para todas las orientaciones) de las semillas dispersadas y de las infecciones establecidas, en relación a la distancia del hospedador focal.

\section{RESULTADOS}

\section{Dispersión de semillas}

El número de semillas dispersadas disminuyó marcadamente con la distancia desde la fuente de semillas $u$ hospedador focal (Fig. 1). La máxima distancia de dispersión primaria registrada desde la fuente de semillas fue de $10 \mathrm{~m}$ hacia el este (Fig. 1). En general, la distancia promedio de dispersión fue de $3.1 \mathrm{~m}$, por lo tanto la mayor acumulación de semillas ocurría cerca de la infección focal. El número de semillas dispersadas en direcciones norte, sur, este y oeste, no mostraron diferencias significativas entre sí (pruebas de Wilcoxon; norte-sur: $\mathrm{P}=0.20, \mathrm{Z}=1.28$; norte-este: $\mathrm{P}=$ $0.22, Z=1.21$; norte-oeste: $P=0.46, Z=0.73$; sur-este: $\mathrm{P}=0.14, Z=1.46$; sur-oste: $\mathrm{P}=0.46, Z$ $=0.73$; este-oste: $\mathrm{P}=0.10, Z=1.62$ ). Las curvas de dispersión, para los cuatro puntos cardinales se ajustaron a un modelo exponencial negativo (Fig. 1). Los valores de los parámetros del modelo y sus ajustes fueron: hacia el norte $\left(\mathrm{y}=2.3+287.48 \mathrm{e}^{-\mathrm{x}}, \mathrm{r}^{2}=\right.$ $\left.0.81, \mathrm{~F}_{(1,9)}=34.8\right)$; $\operatorname{sur}\left(\mathrm{y}=1.76+219.73 \mathrm{e}^{-\mathrm{x}}, \mathrm{r}^{2}=\right.$ $\left.0.84, \mathrm{~F}_{(1,9)}=40.9\right)$, este $\left(\mathrm{y}=2.72+669.56 \mathrm{e}^{-\mathrm{x}}, \mathrm{r}^{2}\right.$ $\left.=0.98, \mathrm{~F}_{(1,9)}=378.4\right)$, oeste $\left(\mathrm{y}=2.04+311.87 \mathrm{e}^{-}\right.$ $\left.\mathrm{x}, \mathrm{r}^{2}=0.95, \mathrm{~F}_{(1,9)}=10.6\right)$ y para el promedio $(\mathrm{y}=$ $\left.1.39+70.75 \mathrm{e}^{-\mathrm{x}}, \mathrm{r}^{2}=0.57, \mathrm{~F}_{(1,9)}=146.0\right)$, donde (x) es la distancia desde las planta madre, e (y) número de semillas dispersadas.

\section{Colonización}

La máxima distancia de colonización desde los arbustos focales fue de $20 \mathrm{~m}$ en la orientación sur Fig. 2. La distancia promedio de colonización de las cuatro orientaciones fue de $13.5 \mathrm{~m}$ (Fig. 2). El número de infecciones de $M$. punctulatum en el sitio de estudio declinó marcadamente con la distancia desde el hospedador focal. Las curvas de colonización hacia cada orientación se ajustaron significativamente a una distribución exponencial negativa (norte: $\mathrm{r}^{2}=0.98$; sur: $\mathrm{r}^{2}=$ 0.98 ; este: $r^{2}=0.99$; oeste: $r^{2}=0.98$ y el promedio de las orientaciones: $r^{2}=0.99$ (Fig. 2)). Las frecuencias de colonización entre orientaciones no presentan diferencias significativas Wilcoxon (norte-sur: $\mathrm{P}=0.50, Z=$ 0.67 ; norte-este: $\mathrm{P}=0.50, Z=0.67$; norte-oeste: $\mathrm{P}=0.79, Z=0.27$; sur-este: $\mathrm{P}=0.60, Z=0.52$; sur-oste: $\mathrm{P}=0.17, Z=1.36$; este-oste: $\mathrm{P}=0.53$, $Z=0.63)$. Las curvas de colonización, en los cuatro puntos cardinales se ajustaron a un modelo exponencial negativo (Fig. 2). Los parámetros de los modelos y sus ajustes fueron: hacia el norte $\left(\mathrm{y}=0.27+408.68 \mathrm{e}^{-\mathrm{x}}\right), \mathrm{r}^{2}=$ $\left.0.98, \mathrm{~F}_{(1,14)}=914.5\right)$, hacia el sur $(\mathrm{y}=0.75+$ $\left.528.61 \mathrm{e}^{\mathrm{e}}, \mathrm{r}^{2}=0.98, \mathrm{~F}_{(1,14)}=619.6\right)$, al este $(\mathrm{y}=$ $\left.0.29+451.75 \mathrm{e}^{-\mathrm{x}}, \mathrm{r}^{2}=0.99, \mathrm{~F}_{(1,14)}=986.4\right)$, al oeste $\left(\mathrm{y}=0.53+497.69 \mathrm{e}^{-\mathrm{x}}, \mathrm{r}^{2}=0.98, \mathrm{~F}_{(1,14)}=\right.$ 663.9) y para el promedio de las orientaciones $\left(\mathrm{y}=0.27+471.07 \mathrm{e}^{-\mathrm{x}}, \mathrm{r}^{2}=0.99, \mathrm{~F}_{(1,14)}=5948.8\right)$, donde (x) es la distancia desde el hospedador, e (y) el número de nuevas infecciones. Respecto a la proporción entre arbustos infectados y no infectados en las distintas orientaciones, se observó que la proporción de individuos infectados fue similar para las orientaciones norte, sur, y este (1:6), y menor que hacia el oeste $(1: 10)$.

\section{Relación entre dispersión y colonización}

Existe una alta correlación entre el número de semillas y el número de infecciones establecidas en función de la distancia desde la fuente de semillas. La correlación de Sperman mostró un alto coeficiente de correlación ( $\mathrm{r}=$ 0.91; $\mathrm{P}<0.01)$. El test de Kolmogorov-Smirnov evidenció que no existen diferencias significativas entre la distribución promedio de la dispersión de semillas y la distribución de las infecciones desde el hospedador focal $(\mathrm{P}>$ 0.10) (Fig. 3).

\section{DISCUSIÓN}

Las distancias de dispersión en Misodendrum punctulatum son acotadas y menores a $10 \mathrm{~m}$. Esto indica que la dispersión de semillas en $M$. punctulatum podría ser una de las más cortas dentro de las plantas hemiparásitas. Incluso, podría ser menor a las hemiparásitas con 

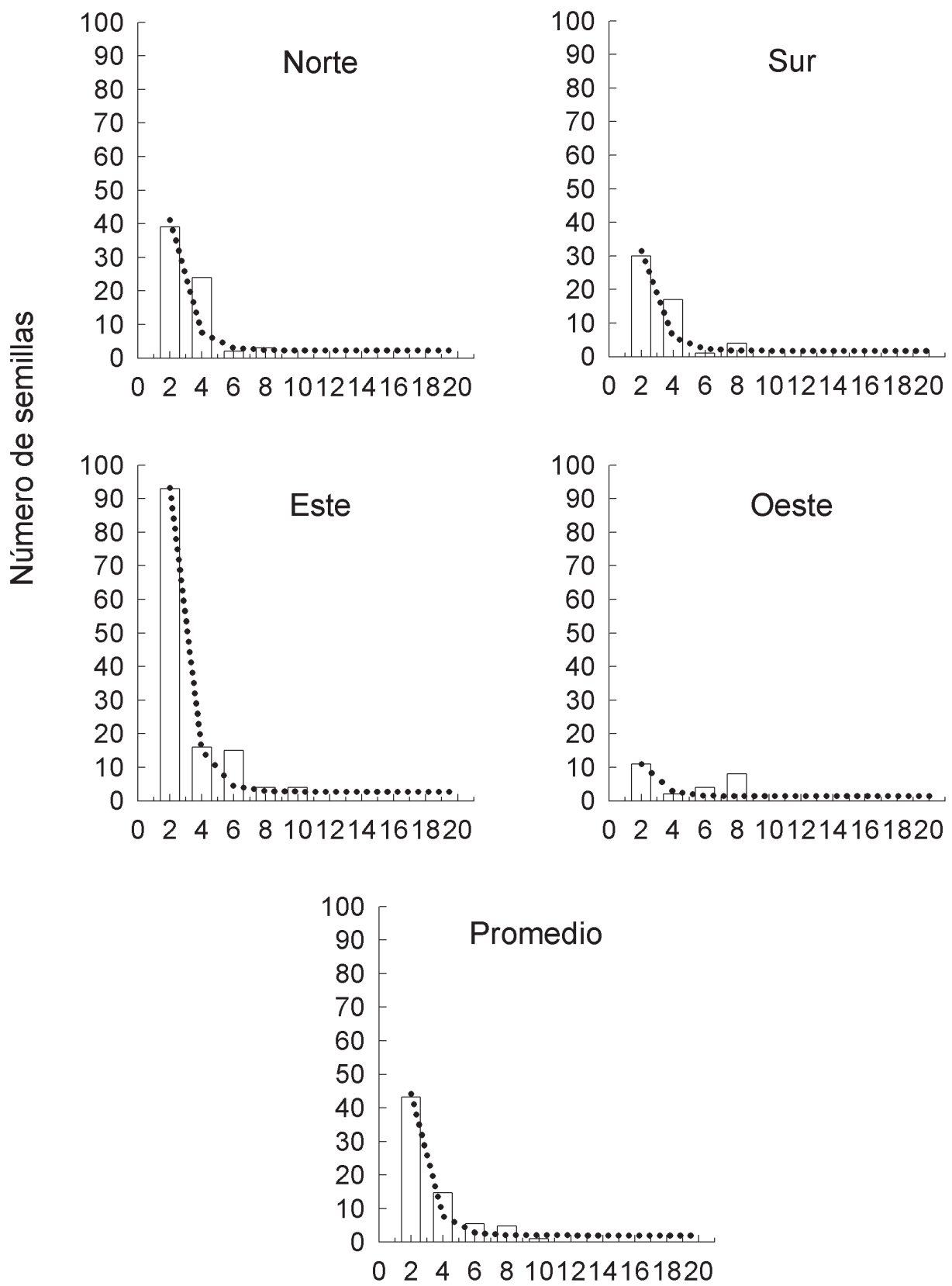

\section{Distancia $(\mathrm{m})$}

Fig. 1: Número promedio de semillas de $M$. punctulatum en diferentes orientaciones en función de la distancia desde la planta madre (barras). Las líneas de puntos indican la distribución (exponencial negativa) a la que se ajustaron los datos de dispersión. Los valores de los parámetros del modelo y sus ajustes fueron: hacia el norte $\left(\mathrm{y}=2.3+287.48 \mathrm{e}^{-\mathrm{x}}, \mathrm{r}^{2}=0.81, \mathrm{~F}_{(1,9)}=34.8\right) ;$ sur $\left(\mathrm{y}=1.76+219.73 \mathrm{e}^{-\mathrm{x}}, \mathrm{r}^{2}=0.84, \mathrm{~F}_{(1,9)}=40.9\right)$, este $\left(\mathrm{y}=2.72+669.56 \mathrm{e}^{-\mathrm{x}}, \mathrm{r}^{2}=0.98, \mathrm{~F}_{(1,9)}=378.4\right)$, oeste $\left(\mathrm{y}=2.04+311.87 \mathrm{e}^{-\mathrm{x}}, \mathrm{r}^{2}=0.95, \mathrm{~F}_{(1,9)}=10.6\right)$ y para el promedio $\left(\mathrm{y}=1.39+70.75 \mathrm{e}^{-\mathrm{x}}, \mathrm{r}^{2}=0.57, \mathrm{~F}_{(1,9)}=146.0\right)$. Donde $(\mathrm{x})$ es la distancia desde las planta madre, $(\mathrm{y})$ número de semillas dispersadas.

Average seeds dispersed of $M$. punctulatum towards different compass aspect from the focal plant (bars). Fitting models for each cardinal point and average (dotted line). Parameters of model fitted were: north $\left(\mathrm{y}=2.3+287.48 \mathrm{e}-\mathrm{x}, \mathrm{r}^{2}=0.81\right.$, $\left.\mathrm{F}_{(1,9)}=34.8\right)$; south $\left(\mathrm{y}=1.76+219.73 \mathrm{e}^{-\mathrm{x}}, \mathrm{r}^{2}=0.84, \mathrm{~F}_{(1,9)}=40.9\right)$, east $\left(\mathrm{y}=2.72+669.56 \mathrm{e}^{-\mathrm{x}}, \mathrm{r}^{2}=0.98, \mathrm{~F}_{(1,9)}=378.4\right)$, west $(\mathrm{y}$ $\left.=2.04+311.87 \mathrm{e}^{-\mathrm{x}}, \mathrm{r}^{2}=0.95, \mathrm{~F}_{(1,9)}=10.6\right)$ and average $\left(\mathrm{y}=1.39+70.75 \mathrm{e}^{-\mathrm{x}}, \mathrm{r}^{2}=0.57, \mathrm{~F}_{(1,9)}=146.0\right)$. ( $\left.\mathrm{x}\right)$ means distance from the focus plant to seeds, and (y) number of seeds dispersed. 

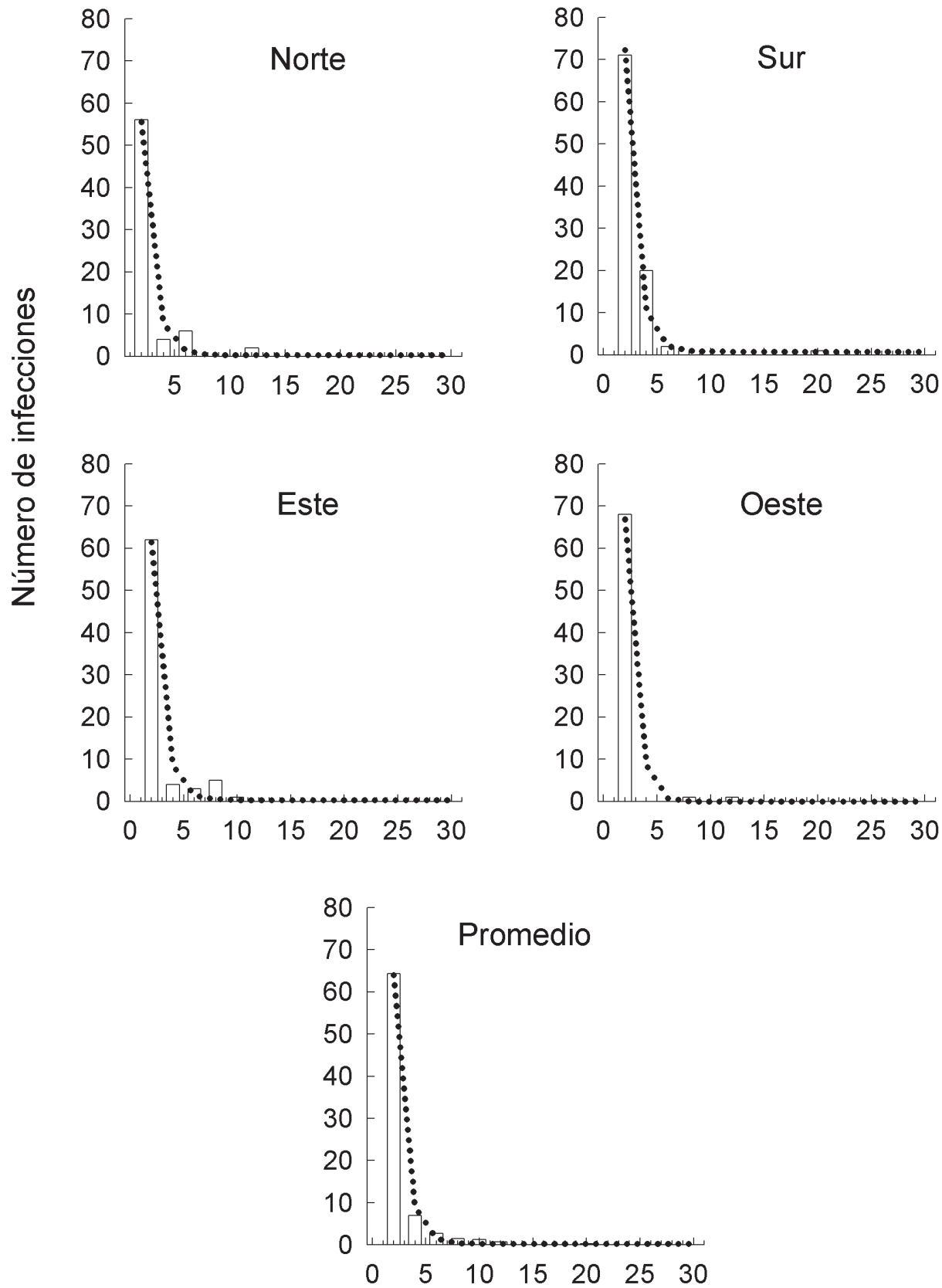

Distancia $(\mathrm{m})$

Fig. 2: Distancia promedio de colonización de M. punctulatum en diferentes orientaciones en función de la distancia desde el hospedador focal (barras). Las líneas de puntos indican la distribución (exponencial negativa) a la que se ajustaron los datos de colonización. Los parámetros de los modelos y sus ajustes fueron: hacia el norte $\left.\left(\mathrm{y}=0.27+408.68 \mathrm{e}^{-\mathrm{x}}\right), \mathrm{r}^{2}=0.98, \mathrm{~F}_{(1,14)}=914.5\right)$, hacia el sur $\left(\mathrm{y}=0.75+528.61 \mathrm{e}^{-\mathrm{x}}, \mathrm{r}^{2}=0.98, \mathrm{~F}_{(1,14)}\right.$ $=619.6)$, al este $\left(\mathrm{y}=0.29+451.75 \mathrm{e} x, \mathrm{r}^{2}=0.99, \mathrm{~F}_{(1,14)}=986.4\right)$, al oeste $\left(\mathrm{y}=0.53+497.69 \mathrm{e}^{-\mathrm{x}}, \mathrm{r}^{2}=0.98, \mathrm{~F}_{(1,14)}=\right.$ $663.9)$ y para el promedio de las orientaciones $\left(\mathrm{y}=0.27+471.07 \mathrm{e}^{-\mathrm{x}}, \mathrm{r}^{2}=0.99, \mathrm{~F}_{(1,14)}=5948.8\right)$. Donde (x) es la distancia desde el hospedador, (y) el número de nuevas infecciones.

Average distance of colonization $M$. punctulatum towards different cardinal directions as a function of the distance to the parent tree (bars). Fitting models for each cardinal point and average (dotted line). Parameters of model fitted were: north $\left.\left(\mathrm{y}=0.27+408.68 \mathrm{e}^{-\mathrm{x}}\right), \mathrm{r}^{2}=0.98, \mathrm{~F}_{(1,14)}=914.5\right)$, south $\left(\mathrm{y}=0.75+528.61 \mathrm{e}^{-\mathrm{x}}, \mathrm{r}^{2}=0.98, \mathrm{~F}_{(1,14)}=619.6\right)$, east $\left(\mathrm{y}=0.29+451.75 \mathrm{e}^{-}\right.$ $\left.\mathrm{x}, \mathrm{r}^{2}=0.99, \mathrm{~F}_{(1,14)}=986.4\right)$, west $\left(\mathrm{y}=0.53+497.69 \mathrm{e}^{-\mathrm{x}}, \mathrm{r}^{2}=0.98, \mathrm{~F}_{(1,14)}=663.9\right)$ and average $\left(\mathrm{y}=0.27+471.07 \mathrm{e}^{-\mathrm{x}}, \mathrm{r}^{2}=0.99\right.$, $\left.\mathrm{F}_{(1,14)}=5948.8\right)$. (x) means distance from the focal host to the new infection, and (y) number of new infection. 


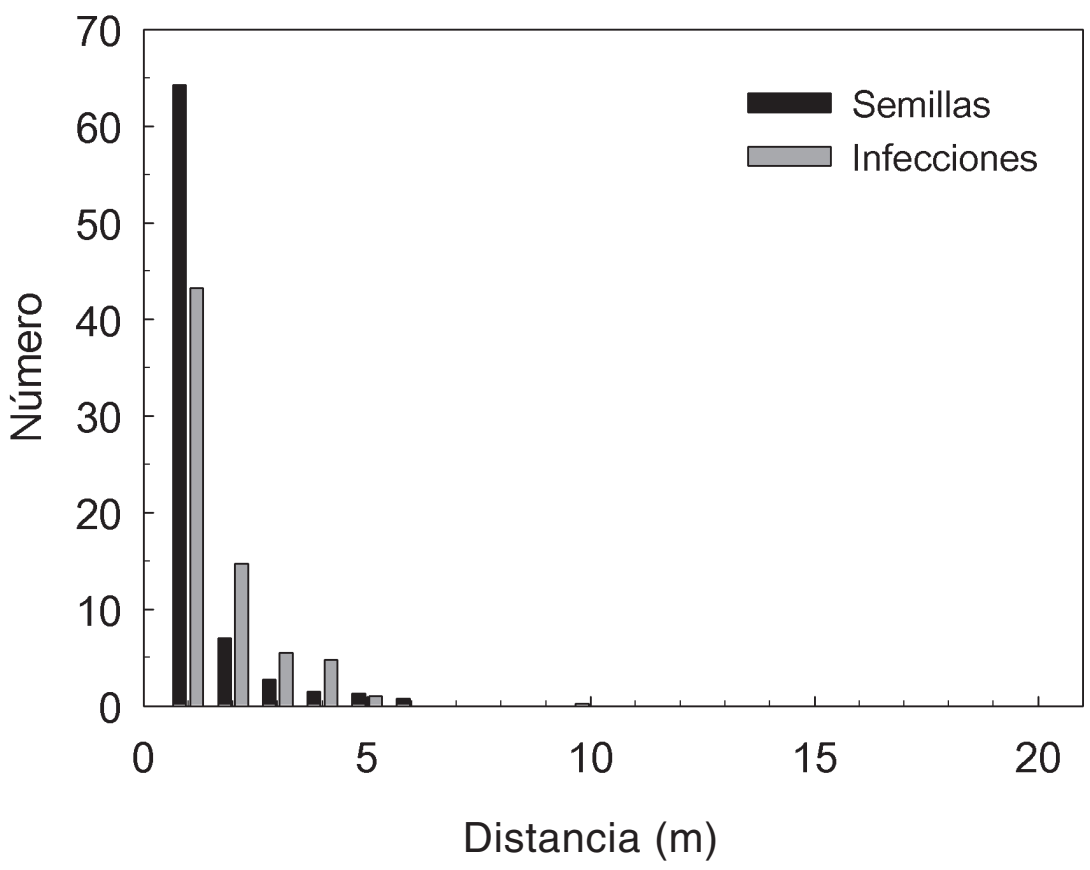

Fig. 3: Distribución promedio de la dispersión de semillas (barras negras) y de las infecciones (barras grises) para cada distancia, desde el hospedador focal.

Average distribution of the seeds dispersal (black bars) and infections (grey bars) by each distances from focal host.

dispersión balística, mecanismo que permite dispersar las semillas hasta $40 \mathrm{~m}$ de distancia desde la fuente (Hawksworth 1961). A diferencia de los muérdagos con dispersión anemócora o balística, los muérdagos dispersados por aves pueden llevar las semillas a más $50 \mathrm{~km}$ desde la planta madre (Murphy et al. 1993).

Las cortas distancias de dispersión en $M$. punctulatum podrían determinar que la velocidad y probabilidad de colonización de nuevos hospedadores sean relativamente bajas. La llegada de las semillas de $M$. punctulatum a un sustrato adecuado para el establecimiento es un proceso fortuito, a diferencia de la mayoría de plantas hemiparásitas dispersadas por aves que cuentan con una relación altamente especializada que ha coevolucionado, convirtiendo a las aves en dispersores eficientes y efectivos (Reid 1991). Las cortas distancias de dispersión de $M$. punctulatum también podrían ser un mecanismo para incrementar la probabilidad de caer sobre los sustratos adecuados para la germinación y el establecimiento y asegurar la supervivencia.
En M. punctulatum la forma de la sombra de las semillas es acotada y asimétrica respecto de la fuente. Esto indica que las semillas se dispersan en su mayoría a cortas distancias de la madre y más lejos hacia algunas orientaciones que hacia otras, en este caso hacia el este, probablemente relacionado con la dirección predominante del viento (noroeste) en el sitio de estudio. Esta misma asimetría también se ha observado en otra especie de los bosques andino patagónicos como Embothrium coccineum (Rovere \& Premoli 2005). Las altas cantidades de semillas encontradas en las ramas que están más cerca de la fuente de semillas, debido a las cortas distancias de dispersión observadas, harían suponer que las setas presentes en los frutos de Misodendrum cumplen un papel más importante como estructuras de anclaje en las ramas de los hospedadores que como estructuras adaptadas para facilitar el vuelo.

Factores como la dirección del viento, disponibilidad de hospedadores, la gravedad y las estructuras de sostén (setas) hacen que las semillas se acumulen en lugares determinados y cerca de la planta madre (en los primeros 4 
m). Durante la dispersión, las semillas se van alejando progresivamente de la fuente tanto vertical como horizontalmente, generando un patrón tridimensional (sensu Willson 1992), que es generado por un efecto de dispersión en cascada. Primeramente se produce una acumulación de semillas en las ramas más cercanas a la fuente, y luego ocurren episodios de dispersión secundaria desde las ramas más altas del hospedador hacia las más bajas. Este patrón hace que las semillas queden dispuestas en un espacio tridimensional con forma de un cono invertido, i.e., que se ensancha a medida que se aleja de la fuente de semillas. La geometría de la trayectoria de dispersión en $M$. punctulatum también puede estar reflejando la distribución de la biomasa de ramas de los hospedadores. En este caso la superficie del cono de dispersión representaría la zona donde están las ramas hospedadoras con menor probabilidad de ser infectadas y la probabilidad de infección incrementaría hacia el interior del cono. Aunque se sabe que en $M$. punctulatum la probabilidad de éxito de infección aumenta con la disminución del diámetro de las ramas (Tercero-Bucardo \& Kitzberger 2004), y los diámetros de las ramas de los hospedadores son determinantes en el éxito de establecimiento de las infecciones de las plantas parásitas (Dawson \& Ehleringer 1991, Sargent 1995, Hawksworth \& Wiens 1996, Amico \& Aizen 2000). La posición y altura de la fuente de semillas, ubicación de la infección en el hospedador son parámetros importantes en la dinámica de infección de $M$. punctulatum. Las semillas que provengan de infecciones que estén a mayor altura podrían alcanzar mayores distancias de dispersión, lo cual incrementa la probabilidad de escapar de la planta focal y colonizar a un nuevo hospedador. El patrón encontrado indicaría que el proceso de recolonización de $M$. punctulatum en poblaciones de hospedadores es lento, e.g. después de un fuego la colonización ocurre a partir de arbustos hospedadores remanentes sobrevivientes a este disturbio. Las primeras infecciones ocurren en proximidades de la fuente de semillas $\mathrm{u}$ hospedador focal y a medida que las infecciones secundarias van madurando sexualmente, estas nuevas infecciones dispersan semillas hacia los hospedadores más lejanos a la fuente primaria. En este trabajo se evidenció una alta correlación espacial entre el número de semillas dispersadas y el número de infecciones, i.e., el mayor número de infecciones se encuentran donde el viento deposita la mayor cantidad de semillas y ocurre en las cercanías del hospedador focal, similar a lo observado por Overton (1994) en el muérdago Phrygilanthus sonorae y Aukema \& Martínez del Río (2002) en Phoradendron californicum. Nuestros resultados también sugieren que en $M$. punctulatum no hay limitación del reclutamiento debido a la dispersión y apoya directamente nuestra hipótesis referida a que la dispersión anemófila de $M$. punctulatum condiciona el patrón de colonización en matorrales postfuego de $N$. antarctica. Si bien la tendencia es pensar que las distancias de dispersión deberían ser mayores que las distancias de colonización, en nuestro caso observamos que la distancia de dispersión y colonización son similares. Esto podría tener dos interpretaciones posibles: que las semillas no son depredadas después de la dispersión y que no ocurren, con frecuencia, eventos de dispersión secundaria.

Si relacionamos las distancias promedio de dispersión de $M$. punctulatum $(3.1 \mathrm{~m})$ con el tamaño promedio de las copas de los hospedadores $(3 \mathrm{~m})$ podríamos inferir que el proceso de reinfección (establecimiento dentro del hospedador que contiene la fuente de semillas) es el hecho más frecuente. Este proceso de reinfección puede facilitar la formación de metapoblaciones de hemiparásitas, donde cada hospedador funciona como un parche de hábitat dentro de una matriz formada por arbustos de especies no hospedadoras $\mathrm{u}$ hospedadores resistentes a dichas infecciones (sensu, Overton 1994). Estudios con otras hemiparásitas han encontrado que existe una relación entre la tasa de infección y el tamaño del hospedador (Elias 1987), debido a que los árboles más grandes recibirán mayor número de semillas. Aunque en otros casos, como en Prygilanthus sonorae, Overton (1994) no encontró resultados consistentes que apoyaran esta relación. En el caso de $M$. punctulatum se ha observado que las altas cargas de parásitas no desencadenan eliminación de ramas de los hospedadores por las infecciones (TerceroBucardo 2001, Tercero-Bucardo \& Kitzberger 2004). De hecho los Nothofagus producen 
mortalidad natural de ramas lo cual no necesariamente está asociado a la carga de infecciones (Veblen et al. 1996).

Un solo trabajo que estudió el efecto fisiológico directo de $M$. punctulatum sobre sus hospedadores, concluye que esta hemiparásitas tiene baja patogenicidad (Tercero-Bucardo 2001). Esta baja patogenicidad podría explicarse por razones de carácter evolutivo, donde se plantea que las características no deletéreas podrían ser una respuesta a las cortas distancias de dispersión de sus semillas (Lipsitch et al. 1995). En este caso, $M$. punctulatum habría desarrollado mecanismos de regulación del uso de agua parecidos a los de sus hospedadores, e.g. control estomático, a fin de mantener una relación con su hospedador durante un tiempo suficiente para colonizar nuevos hospedadores.

Los efectos de las parásitas sobre sus hospedadores también pueden ser indirectos. Se ha observado que al morir las infecciones de $M$. punctulatum los tejidos del hospedador, donde se encontraban los haustorios, son colonizados por hongos xilófagos que producen la descomposición del leño afectado (Cwielong \& Rajchenberg $1995^{2}$, TerceroBucardo 2001), adicionalmente las infecciones debilitan físicamente la rama haciéndola más susceptible a la ruptura por el viento o la nieve. Es decir, la colonización o infección de $M$. punctulatum, sobre $N$. antarctica, no afectaría directamente la supervivencia de la planta hospedadora, pero sí su condición sanitaria, haciéndola más vulnerable al ataque de hongos e insectos xilófagos. La consecuencia más importante de este efecto desde el punto de vista silvicultural es cuando las infecciones ocurren en los troncos principales, ellas generan manchas o pudriciones en el leño bajando la calidad de la madera para su explotación, e.g. en Nothofagus pumilio (Bava 1999). Este mismo autor, encontró que los rodales con alta incidencia de pudriciones de troncos coinciden con los rodales que presentan más infecciones de $M$. punctulatum en los fustes.

2 CWIELONG PP \& RAJCHENBERG M (1995) Forma de ataque y daños provocados por Misodendron punctulatum en la lenga. IV Jornadas Forestales Patagónicas: 56-66, octubre 1995. San Martín de los Andes, Neuquén, Argentina.
Los patrones de distribución espacial de $M$. punctulatum presentados en este trabajo, serán útiles para planificar medidas de manejo con fines comerciales (controlando las infecciones) o de conservación de la biodiversidad. Considerando la acotada dispersión de $M$. punctulatum, una alternativa de manejo en rodales de interés comercial podría basarse en desparasitar los árboles focales para evitar la infección de los renovales. Por otro lado, con fines de conservación, los resultados de este trabajo podrían ser útiles en proyectos de restauración ecológica. En el caso de $N$. antarctica que rebrota en matorrales postfuegos, de no existir individuos remanentes infectados como fuente de semillas se podrían dispersar deliberadamente semillas de $M$. puctulatum en los rebrotes. Los resultados de este trabajo proporcionan un mayor conocimiento de esta interacción ecológica, tan frecuente en los bosques de Nothofagus de Argentina y Chile.

\section{AGRADECIMIENTOS}

Al Consejo Nacional de Investigaciones Científicas y Técnicas, Argentina; Red Latinoamericana de Botánica por subsidio RLB-SP1-99 a NT-B. Universidad Nacional del Comahue y delegación técnica del Parque Nacional Nahuel Huapi. A Guillermo C. Amico por los comentarios sobre el manuscrito.

\section{LITERATURA CITADA}

AGRIOS GN (1969) Plants diseases caused by parasitic plants. En: Agrios GN (ed) Plant patology: 378394. Academic Press, New York, USA.

AIZEN MA \& C EZCURRA (1998) High incidence of plant-animal mutualisms in the woody flora of the temperate forest of southern South America: Biogeographical origin and present ecological significance. Ecología Austral 8: 217-236.

AMICO GC \& MA AIZEN (2000) Mistletoe seed dispersal by a marsupial. Nature 408: 929-930.

AUKEMA JE \& C MARTÍNEZ DEL RIO (2002) Variation in mistletoe seed deposition: Effects of intra- and interspecific host characteristics. Ecography 25: 139-144.

BAVA JO (1999) Aportes ecológicos y silviculturales a la transformación de bosques vírgenes de lenga (Nothofagus pumilio) en bosques manejados en el sector argentino de Tierra del Fuego. CIEFAP, Esquel, Chubut, Argentina.

BUSTAMANTE RO (1996) Depredación de semillas en bosques templados de Chile. En: Armesto JJ, C Villagrán \& MTK Arroyo (eds) Ecología de los bosques nativos de Chile: 265-278. Editorial Universitaria, Santiago, Chile. 
BUSTAMANTE RO \& ML CANALS (1995) Dispersal quality in plants: How to measure efficiency and effectiveness of a seed disperser. Oikos 73: 133136.

CALDER DM (1983) Mistletoes in focus: An introduction. En: Calder DM \& P Bernhardt (eds) The biology of mistletoes: 1-17. Academic Press, New York.

CALDER DM \& P BERNHARDT (eds) (1983) The biology of mistletoes. Academic Press, New York.

DAWSON TE \& JR EHLERINGER (1991) Ecological correlates of seed mass variation in Phoradendron juniperinum, a xylem-tapping mistletoe. Oecologia 85: 332-342.

DE FINA AL (1972) El clima de la región de los bosques Andino-Patagónicos. En: Dimitri M (ed) La región de los bosques Andino-Patagónicos, sinopsis general: 35-58. Instituto Nacional de Tecnología Agropecuaria, Buenos Aires, Argentina.

DONOSO C, L STEINKE \& A PREMOLI (2006) Nothofagus antarctica (G. Forster) Oerst. Nirre, Nire, Niré; Anís (Tierra del Fuego) Ñirre: de Ngërü (mapudungun): zorro. En: Donoso-Zegers C (ed) Las especies arbóreas de los bosques templados de Chile y Argentina. Autoecología. Marisa Cuneo Ediciones, Valdivia, Chile.

DONOSO ZC (ed) (1993) Bosques templados de Chile y Argentina. Variación estructura y dinámica. Editorial Universitaria, Santiago, Chile.

ELIAS P (1987) Chlorophyll contents in leaves of a mistletoe (Loranthus europaeus Jacq.). En: Weber HC \& W Forstreuter (eds) Parasitic Flowering Plants: 171-173. Marburg F.R.G.

GREENE DF \& EA JOHNSON (1989) A model of wind dispersal of winged or plumed seeds. Ecology 70: $339-347$

HARPER JL (ed) (1977) Population biology of plants. Academic Press, London, UK.

HAWKSWORTH FG (1961) Dwarf mistletoe of ponderosa pine in the Southwest. Technical Bulletin 1246. USDA Forest Service, Washington D.C.

HAWKSWORTH FG \& D WIENS (eds) (1996) Dwarf mistletoes: Biology, pathology and systematics. USDA Forest Service, Washington DC.

HERRERA CM, P JORDANO, L LOPEZ-SORIA \& JA AMAT (1994) Recruitment of a mast-fruiting, bird-dispersed tree-bridging frugivore activity and seedling establishment. Ecological Monographs 64: 315-344.

KITZBERGER T (1994) Fire regime variation along a northern Patagonian forest-steppe gradient stand and landscape response. PhD. Thesis. Department of Geography, University of Colorado, Boulder, USA.

KNAPP M, K STÖCKLER, D HAVELL, F DELSUC, F SEBASTIANI \& P LOCKHART (2005) Relaxed molecular clock provides evidence for longdistance dispersal of Nothofagus (southern beech). Public Library of Science Biology 3: 3843.

LAMONT B (1985) Dispersal of the winged fruits of Nuytsia floribunda (Loranthaceae). Australian Journal of Ecology 10: 187-193.

LIPSITCH M, EA HERRE \& MA NOWAK (1995) Host population structure and the evolution of virulence: A "law of diminishing returns". Evolution 49: 743-748.
MCKINNEY LE \& TE HEMMERLY (1977) Host specificity of mistletoe in middle Tennessee III. Davidson County. Journal of the Tennessee Academy of Sciences 52: 40.

MEDEL R, C BOTTO-MAHAN, C SMITH-RAMÍREZ, MA MÉNDEZ, CG OSSA, LN CAPUTO \& WL GONZALES (2002) Historia natural cuantitativa de una relación parásito-hospedero: El sistema Tristerix-cactáceas en Chile semiárido. Revista Chilena de Historia Natural 75: 127-140.

MONTEIRO RF, RP MARTINS \& K YAMAMOTO (1992) Host specificity and seed dispersal of Psittacanthus robustus (Loranthaceae) in South-East Brazil. Journal of Tropical Ecology 8: 307-314.

MURPHY SR, N REID, ZG YAN \& WN VENABLES (1993) Differential passage time of mistletoe fruits through the gut of honeyeaters and flowerpeckers - effects on seedling establishment. Oecologia 93: 171-176.

NATHAN R, UN SAFRIEL \& I NOY-MEIR (2001) Field validation and sensitivity analysis of a mechanistic model for tree seed dispersal by wind. Ecology 82: 374-388.

NORTON DA \& MA CARPENTER (1998) Mistletoes as parasites: Host specificity and speciation. Trends in Ecology and Evolution 13: 101-105.

NORTON DA \& PJ DE LANGE (1999) Host specificity in parasitic mistletoes (Loranthaceae) in New Zealand. Functional Ecology 13: 552-559.

ORFILA EN (1976) Sinopsis de las Misodendraceae de la Argentina y Chile. Revista Facultad Agronomía Universidad La Plata 52: 37-62.

ORFILA EN (ed) (1978) Misodendraceae de la Argentina y Chile. Fundación Elías y Ethel Malamud, Buenos Aires.

OVERTON JM (1994) Dispersal and infection in mistletoe metapopulations. Journal of Ecology 82: 711-723.

OVERTON JM (1997) Host specialization and partial reproductive isolation in desert mistletoe (Phoradendron californicum). Southwestern Naturalist 42: 201-209.

REID N (1989) Dispersal of mistletoes by honeyeaters and flowerpeckers: Components of seed dispersal quality. Ecology 70: 137-145.

REID N (1991) Coevolution of mistletoes and frugivorous birds? Australian Journal of Ecology 16: 457-469.

REID N \& RT LANGE (1988) Host specificity, dispersion and persistence through drought of two arid zone mistletoes. Australian Journal of Botany 36: 299-313.

RESTREPO C, S SARGENT, DJ LEVEY \& DM WATSON (2002) The role of vertebrates in the diversification of New World mistletoes. En: Levey DJ, WR Silva \& M Galetti (eds) Seed dispersal and frugivory: Ecology, evolution and conservation: 83-98. CAB International, Wallingford, UK.

REYES A, M MUÑOZ, H GARCÍA \& C COX (1986) Chemistry of Myzodendraceae, I. Myzodendrome, a new phenylbutanone of Myzodendron punctulatum. Journal of Natural Products 49: 310-320.

RODL T \& D WARD (2002) Host recognition in a desert mistletoe: Early stages of development are influenced by substrate and host origin. Functional Ecology 16: 128-134.

ROSSOW RA (1982) Sinopsis de las Misodendraceae. Parodiana 1: 245-270. 
ROVERE AE \& AC PREMOLI (2005) Dispersión asimétrica de semillas de Embothrium coccineum (Proteaceae) en el bosque templado de Chiloé, Chile. Ecología Austral 15: 1-7.

SARGENT S (1995) Seed fate in a tropical mistletoe: The importance of host twig size. Functional Ecology 9: 197-204.

TERCERO-BUCARDO N (2001) Dinámica de la infección por Misodendrum punctulatum Banks. Ex D.C., en bosques caducifolios de Nothofagus del NO de la patagonia, dispersión, establecimiento y efectos de la hemiparásita, susceptibilidad del hospedador. Tesis de Magíster, Facultad de Agronomía, Universidad de Buenos Aires, Buenos Aires.

TERCERO-BUCARDO N \& T KITZBERGER (2004) Establishment and life history characteristics of the southern South American mistletoe Misodendrum punctulatum (Misodendraceae). Revista Chilena de Historia Natural 77: 509-521.

VEBLEN TT, C DONOSO, T KITZBERGER \& AJ REBERTUS (1996) Ecology of Southern Chilean

Editor Asociado: Mario George-Nascimento

Recibido el 6 de julio de 2009; aceptado el 27 de abril de 2010 and Argentinian Nothofagus forests. En: Veblen TT, RS Hill \& J Read (eds) The ecology and biogeography of Nothofagus Forests: 293-353. Yale University Press, New Haven.

VEBLEN TT \& DC LORENZ (1987) Post-fire stand development of Austrocedrus-Nothofagus forests in northern Patagonia. Vegetatio 71: 113-126.

VIDAL-RUSSELL R \& A PREMOLI (2004) Variación en Nothofagus antarctica (Forst) Oerst. En: Donoso C, A Premoli, L Gallo \& R Ipinza (eds) Variación intraespecífica en las especies arbóreas de los bosques templados de Chile y Argentina: 173188. Editorial Universitaria, Universidad Austral de Chile, Valdivia, Chile.

WANG BC \& TB SMITH (2002) Closing the seed dispersal loop. Trends in Ecology and Evolution 17: 379-385.

WILLSON MF (1992) The ecology of seed dispersal. En: Fenner M (ed) The ecology of regeneration in plant communities: 61-85. Cab International. Department of Biology, University of Southampton, Southampton, UK. 